\title{
Breast Arterial Calcifications on Mammography Do Not Predict Myocardial Ischemia on Myocardial Perfusion Single-Photon Emission Computed Tomography
}

\author{
Ahmed Fathala ${ }^{a}$ c c, Salma Salem ${ }^{a}$, Fahad Alanazi $^{a}$, Deema Abunayyan ${ }^{\mathrm{a}}$, \\ Abdelmoneim M. Eldali ${ }^{\mathrm{b}}$, Abdulaziz Alsugair ${ }^{\mathrm{a}}$
}

\begin{abstract}
Background: The aim of this study was to determine if breast arterial calcification (BAC) on mammography predicts myocardial ischemia (MI) on stress myocardial perfusion single-photon emission computed tomography (MPS). BAC is a type of medial artery calcification that can be seen incidentally on mammography, but the relationship between coronary artery calcification and MI on MPS is yet unknown.
\end{abstract}

Methods: A total of 435 consecutive women underwent mammography and stress MPS within 1 year of each other. BAC was quantitatively evaluated $(0-13)$. Patients with known coronary artery diseases (CADs) such as coronary artery bypass grafting (CABG), percutaneous coronary intervention (PCI), MI, positive coronary angiogram or positive MPS were excluded from the study. Risk factors for $\mathrm{CAD}$ were obtained from a chart review.

Results: The mean age was $58 \pm 8$ years. BAC was found in 258 $(59 \%)$ of the study population. BAC-positive patients were significantly older than BAC-negative patients $(\mathrm{P}<0.0001)$, there were strong associations between $\mathrm{BAC}$ and hypertension $(\mathrm{P}=0.0309)$, chronic kidney disease $(\mathrm{CKD})(\mathrm{P}=0.0001)$, and diabetes $(\mathrm{P}=$ $0.0309)$, but there were significant associations between BACV and hyperlipidemia, family history of CAD, and smoking $(\mathrm{P}=0.6856, \mathrm{P}$ $=0.9642$, and $\mathrm{P}=0.087$, respectively). The mean score of BAC was $5 \pm 5$ in patients with normal MPS and was $6 \pm 6$ in patients with abnormal MPS. There were no associations between total BAC and MPS results $(\mathrm{P}=0.2095)$, and between BAC categories and MPS result $(\mathrm{P}=0.3069)$.

Manuscript submitted September 5, 2017, accepted September 27, 2017

aDepartment of Radiology, Nuclear Medicine and Cardiovascular Imaging, King Faisal Specialist Hospital \& Research Center, PO Box 3354, Riyadh, Saudi Arabia

${ }^{b}$ Research Centre, Department of Biostatistics, Epidemiology and Scientific Computing, King Faisal Specialist Hospital \& Research Center, PO Box 3354 Riyadh, Saudi Arabia

${ }^{\mathrm{c} C o r r e s p o n d i n g ~ A u t h o r: ~ A h m e d ~ F a t h a l a, ~ M e d i c a l ~ I m a g i n g ~ S e r v i c e / D e p a r t-~}$ ment of Radiology, King Faisal Specialist Hospital and Research Center, MBC\#28, PO Box 3354, Riyadh, Saudi Arabia.

Email:ahm35799@hotmail.com

doi: https://doi.org/10.14740/cr604w
Conclusions: Based on our study, the presence and severity of BAC on screening or diagnostic mammography do not predict MI on stress MPS, and further cardiac workup based on the presence of BAC is not warranted. BAC is very common in mammography up to $59 \%$ and associated with age, diabetes, $\mathrm{CKD}$, and hypertension. In contrast, the prevalence of MI is only $13 \%$ in women with BAC and associated with age, diabetes, CKD, hyperlipidemia, and impaired left ventricular function.

Keywords: Breast calcification; Myocrdial ischmeia; CAD

\section{Introduction}

Breast arterial calcification (BAC) is a type of medial artery calcification that can be seen incidentally on mammography, and deposition of calcification in the arterial media is known as arteriosclerosis which is also known as Monckeberg's arteriosclerosis [1,2]. The deposition of calcified and non-calcified plaques in the arterial intima is known as atherosclerosis [3]. However, some recent studies suggested that Monckeberg's arteriosclerosis involves both the media and internal elastic lamina [4]. Pathological studies demonstrated that the presence of medial calcification in the aorta and arteries of lower extremities is more common in the older population [5] and other selected groups such as diabetes mellitus [6] and renal disease patients [7]. Recently, many investigations have demonstrated that BAC is a potential women-specific risk factor for both coronary artery disease (CAD) and cardiovascular disease (CVD) [8]. Unfortunately, the presence or absence of $\mathrm{BAC}$ is often ignored during the mammographic assessment, and several studies have demonstrated that the presence and severity of subclinical atherosclerosis and CVD could be assessed by mammograms $[9,10]$. The presence of coronary artery calcification (CAC) is a well-established risk factor for $\mathrm{CAD}$, and studies have demonstrated that higher coronary artery calcium score (CACS) is associated with higher risk of coronary heart disease events [11], and the presence of CAC has also been used to justify the use of more aggressive CAD risk factor modification treatment [12]. The relationship between $\mathrm{BAC}$ and $\mathrm{CAC}$ has been examined in several studies; the majority of published studies point to a significant relationship between $\mathrm{BAC}$ and $\mathrm{CAC}$, and the presence of $\mathrm{BAC}$ 


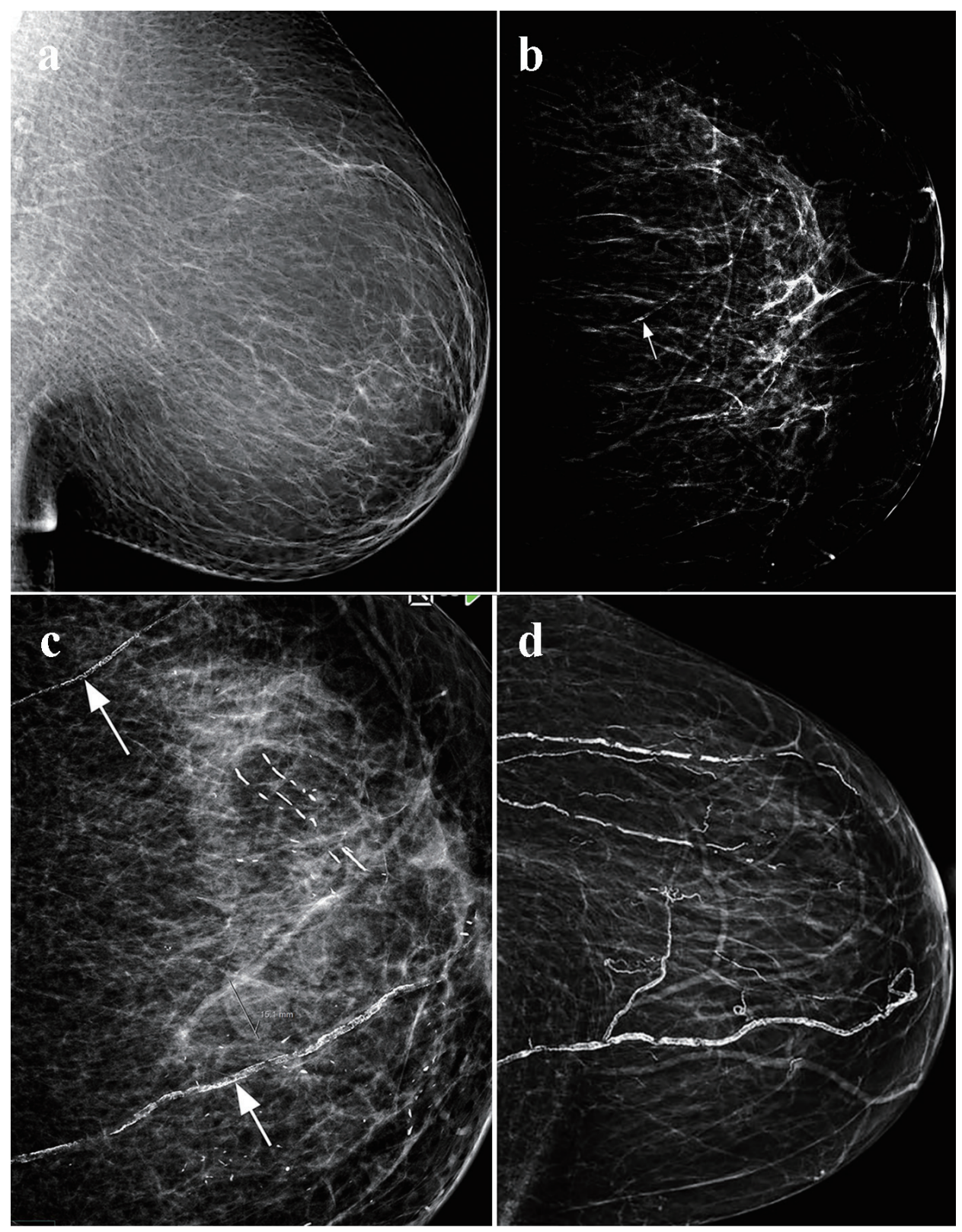

Figure 1. Visual scoring of breast arterial calcification (BAC): (a) no BAC (score 0); (b) mild BAC, one vessle (arrow), single wall, and less than one-third of the vessel length (score 3); (c) moderate BAC: two vessels (arrows), two wall with visualization of the vessels lumen and less than two-thirds of the vessel length (score 8); (d) severe BAC: multiple vessels, dense calcification with obliteration of the vessels lumen, and more than two-thirds of the vessels length (score 13).

improved the ability to identify women with CAC compared to Framingham risk score (FRS) alone [13-18]. Myocardial perfusion single-photon emission computed tomography (MPS) plays a key role in diagnosing CAD, and establishing prognosis and effectiveness of therapy $[19,20]$. Prior studies have demonstrated the strong relationship between presence or absence of MI on MPS and CACS [21, 22]. However, currently, there are no studies investigating the relationship between BAC and MPS, and this study aimed to determine if breast calcification on mammogram could determine the presence of MI in MPS.

\section{Materials and Methods}

\section{Study population}

This retrospective study was approved by the institutional review board at our institution. A total of 435 females, however, underwent screening or diagnostic mammogram and stress MPS for clinically appropriate indications from January 2015 to December 2016, and both mammogram and MPS were performed within 1 year of each other. Patients with known CADs 
Table 1. Patient Characteristics and Comparison of Presence and Absence of BAC Based on CAD Risk Factors

\begin{tabular}{lllll}
\hline & All & Absence of BAC & Presence of BAC & P-value \\
\hline $\mathrm{N}$ & 435 & $177(41 \%)$ & $258(95 \%)$ & 0.0001 \\
Age, years (mean \pm SD) & $58 \pm 8$ & $55 \pm 7$ & $61 \pm 8$ & 0.0148 \\
Diabets mellitus & 237 & 84 & 183 & 0.0309 \\
Hyprtension & 291 & 108 & 41 & 0.087 \\
Smoking & 2 & 2 & 0 & 0.6856 \\
Hyerlipidemia & 194 & 81 & 113 & 0.0001 \\
Chronic kidney disease & 176 & 49 & 127 & 0.9642 \\
Family history & 10 & 4 & 6 & \\
\hline
\end{tabular}

such as coronary artery bypass grafting (CABG), percutaneous coronary intervention (PCI), MI, prior positive coronary angiogram or positive MPS were excluded from the study. Risk factors for CAD were obtained from a chart review.

\section{MPS acquisition and analysis}

Stress and rest single-photon emission computed tomography (SPECT) imaging were performed with technetium-99m tetrofosmin according to standard American Society of Nuclear Cardiology guidelines [23]. Most subjects underwent pharmacological stress test, with dipyridamole using standard infusion protocols. Medications such as nitrates and beta-blockers were stopped at least $12 \mathrm{~h}$ before testing. The SPECT images were visually interpreted in all three standard projections, along with the gated SPECT and raw image data to assess for perfusion. All images were re-oriented in short, vertical, and horizontal views, utilizing auto-SPECT (Cedars-Sinai Medical Center, Los Angeles, CA) for visual interpretation by an experienced nuclear medicine physician. The reader was not biased by clinical information. Stress and rest perfusion images were scored using 17 tomographic segments, which included six segments each for the basal and mid-ventricular slices, and four segments for the apical short-axis slices. The final segment was located in the most apical part of the left ventricle. Finally, gated short-axis images were processed with quantitative SPECT software, to measure the ejection fraction. In the visual analysis, the 17 segments were scored for perfusion defects on a four-point system $(0=$ normal; $1=$ mild; $2=$ moderate; and 3 = severe), for both the stress and rest images. A gated SPECT result was considered normal if as follows: no visual perfusion defect, summed stress score $<3$, a left ventricular ejection fraction (LVEF) at rest $>50 \%$.

\section{Mammography}

Mammograms were performed using full-field digital mammography system on either Hologic Dimensions unit (Bedford, MA) or GE Senographe Essential (GE, Paris, France), with the acquisition of standard views. An experienced observer interpreted the mammogram on a dedicated, calibrated workstation in a blinded fashion. BAC scoring was reported as described previously by Margolies et al [18], with some modification. The density of calcium in the most severely affected artery was reordered as none (score 0 ); mild, only one vessel wall involved (score 1); moderate, two vessel walls were involved (score 2); and severe, with severe calcification and complete obliteration of the vessels wall (score 3 ). The length of the involved vessel was recorded as none (score 0 ), less than one-third of the vessels (score 1), one-third to two-thirds (score 2), and more than two-thirds of vessel length (score 3 ). The number of the involved vessels in each breast was numerically coded as 1 to 6 ; in the case where more than six vessels are involved, the maximum coded number was 6 , with total score ranging from 0 to 13 . The total score was further grouped into four categories: zero BAC (none), mild BAC (score 1 - 4), moderate (5 - 8), and severe (9 - 13) (Fig. 1a, b, c, and d).

\section{Statistical analysis}

The software package SAS version 9.4 (SAS Institute Inc., Cary, NC, USA) was used to analyze the data of this retrospective study. Descriptive statistics for the continuous variables were reported as mean \pm standard deviation and categorical variables were summarized as frequencies and percentages. Continuous variables were compared using Student's $t$-test and Pearson's correlation test, while categorical variables were compared using Chi-square test. Univariate and multivariate logistic regression analyses were used to define which demographic and clinical characteristics are associated with the main outcomes of the study. The level of statistical significance is set at $\mathrm{P}<0.05$.

\section{Results}

\section{Patient characteristics and comparison of presence and ab-} sence of BAC based on CAD risk factors

BAC was found in $258(59 \%)$ of the study population (Table 1). BAC-positive patients were significantly older than BACnegative patients $(\mathrm{P}<0.0001)$, there were strong associations between $\mathrm{BAC}$ and hypertension $(\mathrm{P}=0.0309)$, chronic kidney disease $(\mathrm{CKD})(\mathrm{P}=0.0001)$, and diabetes $(\mathrm{P}=0.0309)$, but 
Table 2. Comparison of Normal MPS and Abnormal MPS Based on CAD Risk Factors

\begin{tabular}{|c|c|c|c|c|}
\hline & All & Normal MPS, 380 (87\%) & Abnormal MPS, 55 (13\%) & P-value \\
\hline $\mathrm{N}$ & 435 & $380(87 \%)$ & $55(13 \%)$ & \\
\hline Absence of BAC & 177 & $155(36 \%)$ & $22(5 \%)$ & \\
\hline Diabetes mellitus & 237 & 199 & 38 & 0.0199 \\
\hline Hypertension & 291 & 250 & 41 & 0.1971 \\
\hline Hyperlipidemia & 194 & 162 & 32 & 0.0301 \\
\hline Chronic kidney disease & 176 & 145 & 31 & 0.0101 \\
\hline Family history & 10 & 9 & 1 & 0.7991 \\
\hline
\end{tabular}

there were significant associations between BACV and hyperlipidemia, family history of CAD, and smoking $(\mathrm{P}=0.6856, \mathrm{P}$ $=0.9642$, and $\mathrm{P}=0.087$, respectively).

\section{Comparison of normal MPS and abnormal MPS based on CAD risk factors}

Normal MPS was found in $380(87 \%)$ of the population and abnormal in only $13 \%$. There was no association between MPS and BAC with P value of 0.3069 (Table 2). There were associations between MPS and diabetes $(\mathrm{P}=0.0199)$, hyperlipidemia $(P=0.0301)$, and CKD $(P=0.0101)$. There were no associations between MPS and hypertension, smoking, and family history of CAD $(\mathrm{P}=0.1971, \mathrm{P}=0.111$, and $\mathrm{P}=0.7991$, respectively).

\section{Comparison of normal MPS and abnormal MPS based on the age and left ventricular function}

The mean age of the total study population was $58 \pm 8$ years, and the mean left ventricular function was $63 \pm 9 \%$ (Table $3)$. There was a strong association between MPS and patient age and left ventricular function with $\mathrm{P}$ values of 0.0001 and 0.0425 , respectively.

Relationship between BAC was based on the number of the arteries, density of calcification, and length of the vessels, and total BAC score and MPS.

The number of involved breast arteries was mostly one $(19 \%)$, two $(12 \%)$, and three $(29 \%)$, but more than three arteries were less than $1 \%$. The distribution of the density of calcification and length of the vessels were distributed more equally among all patients with positive BAC. The mean score of BAC was $5 \pm 5$ in patients with normal MPS and was $6 \pm$ 6 in patients with abnormal MPS. There were no associations between the number of the involved arteries, density of calcification, length of the involved vessels, and total BAC and MPS results $(\mathrm{P}=0.3668, \mathrm{P}=0.1693, \mathrm{P}=0.4954$, and $\mathrm{P}=0.2095$, respectively) (Table 4$)$.

\section{Relationship between BAC score categories and MPS}

BAC score categories include zero group (absence of BAC), mild (1 - 4), moderate (5 - 8), and severe (9 - 13); total number of zero category was 177 , mild category was 46 , moderate category was 67 , and severe category 143 . There was no association between BAC categories and MPS result $(\mathrm{P}=0.3069)$ (Table 5).

\section{Predicting abnormal MPS by multivariate analysis}

Multivariable logistic regression analysis revealed that the left ventricular function was the most potent predictor for MPS ischemia in our study; additional significant variables include age and CKD. However, diabetes and hyperlipidemia were not predictors on multivariate analysis (Table 6).

\section{Discussion}

This study is the first to demonstrate that there is no association between semi-quantitative BAC mammography and MI on MPS in a large female cohort. Furthermore, there is no association between different BAC variables such as number of breast arterial involvement, density of calcification, length of

Table 3. Comparison of Normal MPS and Abnormal MPS Based on the Age and Left Ventricular Function

\begin{tabular}{lllll}
\hline & All & Normal MPS, 380 & Abnormal MPS & P-value \\
\hline $\mathrm{N}$ & 435 & $380(87 \%)$ & $55(13 \%)$ & 0.0425 \\
Age, years (mean \pm SD) & $58 \pm 8$ & $58 \pm 8$ & $60 \pm 8$ & 0.0001 \\
Left ventricular function, \% (mean \pm SD) & $63 \pm 9$ & $64 \pm 8$ & $57 \pm 12$ & \\
\hline
\end{tabular}


Table 4. Relationship Between Breast Arterial Calcification Based on the Number of the Arteries, Density of Calcification, and Length of the Vessels, and Total BAC Score and MPS

\begin{tabular}{|c|c|c|}
\hline & Number (\%) & P-value \\
\hline Number of involved breast arteries & & 0.3668 \\
\hline 0 & $177(41 \%)$ & \\
\hline 1 & $81(19 \%)$ & \\
\hline 2 & $50(12 \%)$ & \\
\hline 3 & $124(29 \%)$ & \\
\hline 4 & $1(0.2 \%)$ & \\
\hline 5 & $1(0.2 \%)$ & \\
\hline 6 & $1(0.2 \%)$ & \\
\hline Density of calcification & & 0.1693 \\
\hline 0 & $177(40)$ & \\
\hline 1 & $94(22 \%)$ & \\
\hline 2 & $55(13 \%)$ & \\
\hline 3 & $57(13 \%)$ & \\
\hline 4 & $53(12 \%)$ & \\
\hline Length of vessels & & 0.4954 \\
\hline 0 & $177(41 \%)$ & \\
\hline 1 & $81(19 \%)$ & \\
\hline 2 & $50(12 \%)$ & \\
\hline 3 & $126(28 \%)$ & \\
\hline Total score of arterial calcification & & 0.2095 \\
\hline Patient with normal MPS (mean \pm SD) & $5 \pm 5$ & \\
\hline Patient with abnormal MPS (mean \pm SD) & $6 \pm 6$ & \\
\hline
\end{tabular}

the vessels and MI. The study demonstrated that there are multiple predictors of MI, including patient age, diabetes, hyperlipidemia, CKD, and left ventricular function, in contrast to the predictor BAC that includes patient age, diabetes and CKD.

\section{Prior studies}

To the best of our knowledge, no prior studies reported the relationship between BAC detected on mammography and stress MPS or other MI detection techniques such as stress echocardiography or stress magnetic resonance imaging. But there is one study that reported the relationship between BAC and conventional coronary angiography and a few retrospective

Table 5. Relationship Between BAC Score Categories and MPS

\begin{tabular}{llll}
\hline BAC score category & Normal MPS & Abnormal MPS & P-value \\
\hline Zero & $155(36 \%)$ & $22(5 \%)$ & \\
Mild $(1-4)$ & $45(6 \%)$ & $1(\%)$ & \\
Moderate $(5-8)$ & $60(14 \%)$ & $7(2 \%)$ & \\
Severe $(9-13)$ & $120(28 \%)$ & $23(5 \%)$ & 0.3069 \\
\hline
\end{tabular}

studies investigated the relationship between BAC and CAC. Zgheib et al examined the relationship between BAC and coronary angiography in 172 women. The presence of BAC was noted and compared with the presence of CAD and presence of CAD risk factors. The authors did not observe a correlation between BAC and coronary angiography detected CAD, even when CAD severity was considered. This finding is highly concurrent with our main finding that the presence of BAC does not necessarily indicate flow limiting lesion on coronary angiography or MI on stress MPS [24]. In contrast, the relationship between BAC and CAC has been examined in several small studies. Margolies et al, retrospectively, examined 292 women with digital mammography and non-gated computed tomography (CT). BAC was quantitatively evaluated $(0-12)$

Table 6. Multivariate Predictors of Abnormal MPS Result

\begin{tabular}{ll} 
& P-value \\
\hline Age (years) & 0.0342 \\
Diabetes & 0.3081 \\
Hyperlipidemia & 0.1541 \\
Chronic kidney disease & 0.0778 \\
Mean left ventricular function & $<0.0001$
\end{tabular}


and CAC was visually estimated in CT. The authors found a strong association of BAC and CAC. BAC was found to be superior to standard cardiovascular risk factors, and high statistical association between total graded BAC score, number of calcified vessels, density and length of the vessels involved and CAC score (all P values < 0.0001) [18]. Pecchi et al prospectively examined 74 Italian postmenopausal women aged more than 65 years without CAD and found a modest correlation of $\mathrm{BAC}$ with the presence of CAC as seen on CT. Interestingly, in this study, the prevalence of BAC was $59 \%$ which is similar to study population [13]. More recently, Newallo et al examined a group of 204 African-American women who had mammography and also referred for coronary computed tomography angiography (CCTA) and CACS, and the authors retrospectively reviewed both imaging studies for the presence of $\mathrm{BAC}$ and CAD. The BAC prevalence was only $21 \%$ with CAC more than 100 in 24 of the 204 subjects. Women with BAC-positive were seven times more likely to have CAC higher than 100, compared with women without BAC [16]. In another study, Chadashvili et al performed a retrospective analysis of 145 women referred for CCTA within 1 year of mammography, and found an association of BAC presence with CACS more than 11. The presence of BAC correlates with more than $25 \%$ for developing $\mathrm{CAD}$. In the BAC-positive group, $70 \%$ of subjects had more than $25 \%$ compared with only $45 \%$ in the BAC-negative group [17]. In contrast, Moradi et al examined 150 women aged more than 40 years and CCTA; the authors found that there was no significant difference between patients with different severity of BAC and CAC score [25]. With the exception of Morardi study, the published studies point to a significant relationship between BAC and CAC. However, these data should be interpreted with caution because of small sample sizes with possible selection bias, different methods for measuring $\mathrm{CAC}$ and $\mathrm{BAC}$, and different ethnic groups.

\section{Impact of $\mathrm{CAD}$ risk factors on $\mathrm{MI}$ and $\mathrm{BAC}$}

Although the study data showed no significant association between BAC and ischemia on MPS, there are several traditional risk factors associated with ischemia on MPS, including age, diabetes, hyperlipidemia, CKD, and left ventricular function. In multivariate logistic regression analysis, the most potent predictors for ischemia were left ventricular function $(\mathrm{P}<0.0001)$ and age $(\mathrm{P}=0.0342)$. The prevalence of $\mathrm{MI}$ was $13 \%$ in the study population. We do not have coronary angiography correlation for those patients with abnormal MPS. The possibility of false positive studies cannot be completely excluded. In female patients, breast attenuation is common and may result in a perfusion defect along anterior wall of the left ventricle. It sometimes can be difficult to distinguish breast attenuation form true defects, and in our laboratory we utilize attenuation correction and gated SPECT which greatly improve the specificity and we had excellent correlation with coronary angiography. On the other hand, the main predictors for BAC on mammography were age, diabetes, and CKD. The location of BAC and CAC is different: BAC is the manifestation of Monckeberg's arteriosclerosis also knows as calcific medial sclerosis but CAC is the marker of atherosclerosis. Our current study and other prior studies demonstrated significant consistent association for age, CKD, diabetes, and hypertension with no relationship to the conventional CAD risk factors for hyperlipidemia, smoking, or family history of CAD. However, the lack of association between BAC and MI as demonstrated in our study, and lack of correlation between BAC and obstructive coronary artery disease on coronary angiography, explained that both calcific medial sclerosis and arterial atherosclerosis share most CAD risk factors, such as age, diabetes, CKD, a discrepancy between anatomy (as shown by CAC), and physiology (MI on MPS), or may be different mechanism that need further studies. For example, patients with CKD have high incidence and severity of CAD compared to patients with normal renal function [26], standard risk factors for CAD are common in CKD, oxidative stress has been linked to the pathogenesis of plaques formation and plaques rupture [27]. Arterial medial calcification was found to be a strong prognostic marker of all cause and CV mortality in hemodialysis patients, independent of conventional CAD risk factors. The principal effect of arterial medial calcification on arterial function is increased arterial stiffness [7].

\section{Prevalence and visual scoring of BAC}

The prevalence of BAC in our study population is $59 \%$, which is significantly higher compared to previous published data. The prevalence of BAC in published studies varies from $1 \%$ to $49 \%$. Age is the most powerful factor influencing the prevalence of BAC (sickles). Race and ethnicity is another significant factor in the prevalence of BAC. In a large cohort of women aged 32 - 92 years who had routine mammography, Hispanic women had highest prevalence (34\%), whereas Asian women had the lowest (7\%), vs. $24 \%$ in white women, and $25 \%$ in African women [28]. Pecchi et al examined 74 postmenopausal women aged more than 65 years and found a high prevalence of BAC up to $59 \%$, which is exactly similar to the prevalence of BAC in our study. Based on these data, it appears that beside the age, the race ethnicity, geographical location also determines the prevalence and severity of BAC [13]. Further studies are needed for better understanding of BAC prevalence and its key determinants in multi-ethnic, international populations. Ordinal BAC score was descried by Margolies et al [18]; however, we modified the visual scoring, instead of maximum score of 12 to score of 13 . The density calcium in the most severely affected segment was recorded as none (score 0 ); mild, only one vessel wall involved (score 1); moderate, two vessel wall involved (3); and severe, with no visible lumen (4). There are quite a number of variabilities in reporting BAC on mammography. One of the previous studies by Henkin et al reported BAC as the presence of linear deposits of calcifications along the periphery of tapered structures, which is the typical configuration of the breast vessels but with no visual scoring [29]. In another study by Zgheib et al, BAC status was determined by defining discrete BAC variable with no scoring system, and BAC was defined as a dichotomous variable, total number of quadrant containing BAC, and longest continuous variable [24]. The authors of the previous studies investigating $\mathrm{BAC}$ and $\mathrm{CAD}$ have used different scoring and visualization 
techniques; obviously, lack of standardization of BAC reporting on mammography is an important limitation of previous studies which limits accurate comparison among different reports. The ideal scoring system to report BAC requires further studies and investigations.

\section{Study limitations}

We acknowledge that our study has some limitations. This is a retrospective study of women who had mammography and were referred for clinically indicated stress MPS, and therefore, subjected to selection bias, including coexisting and/or high prevalence of CAD for which MPS was ordered. Ordinal scoring of BAC was not validated or reported in other published study, although we have applied a scoring system very similar to published BAC score by Margolies et al [18], further investigation and studies are required to standardize BAC reporting on mammography. Since women with positive MPS did not undergo coronary angiography, there is a possibility of false positive MPS and is not excluded particularly in women with breast attenuation artifacts. Finally, the study was conducted on Middle Eastern, Mediterranean population with different ethnic, racial, and social background; caution must be exercised in applying the result to the worldwide population.

In summary, based on our current study, the presence and severity of BAC on screening or diagnostic mammography do not predict MI on stress MPS, and further cardiac workup based on the presence of BAC is not warranted. BAC is very common on mammography up to $59 \%$, and associated with age, diabetes, $\mathrm{CKD}$, and hypertension. In contrast, the prevalence of MI is only $13 \%$ in women with BAC and associated with age, diabetes, CKD, hyperlipidemia, and impaired left ventricular function. Probably this group of women will benefit with additional cardiac workup for better diagnosis and stratification of CAD.

\section{References}

1. Everhart JE, Pettitt DJ, Knowler WC, Rose FA, Bennett PH. Medial arterial calcification and its association with mortality and complications of diabetes. Diabetologia. 1988;31(1):16-23.

2. Shanahan CM, Cary NR, Salisbury JR, Proudfoot D, Weissberg PL, Edmonds ME. Medial localization of mineralization-regulating proteins in association with Monckeberg's sclerosis: evidence for smooth muscle cell-mediated vascular calcification. Circulation. 1999;100(21):2168-2176.

3. Trion A, van der Laarse A. Vascular smooth muscle cells and calcification in atherosclerosis. Am Heart J. 2004;147(5):808-814.

4. Micheletti RG, Fishbein GA, Currier JS, Fishbein MC. Monckeberg sclerosis revisited: a clarification of the histologic definition of Monckeberg sclerosis. Arch Pathol Lab Med. 2008;132(1):43-47.

5. Blumenthal HT, Lansing AI, Wheeler PA. Calcification of the media of the human aorta and its relation to in- timal arteriosclerosis, ageing and disease. Am J Pathol. 1944;20(4):665-687.

6. Lehto S, Niskanen L, Suhonen M, Ronnemaa T, Laakso M. Medial artery calcification. A neglected harbinger of cardiovascular complications in non-insulin-dependent diabetes mellitus. Arterioscler Thromb Vasc Biol. 1996;16(8):978-983.

7. London GM, Guerin AP, Marchais SJ, Metivier F, Pannier $\mathrm{B}$, Adda $\mathrm{H}$. Arterial media calcification in end-stage renal disease: impact on all-cause and cardiovascular mortality. Nephrol Dial Transplant. 2003;18(9):1731-1740.

8. Jiang X, Clark M, Singh RK, Juhn A, Schnatz PF. Association of breast arterial calcification with stroke and angiographically proven coronary artery disease: a metaanalysis. Menopause. 2015;22(2):136-143.

9. Hendriks EJ, de Jong PA, van der Graaf Y, Mali WP, van der Schouw YT, Beulens JW. Breast arterial calcifications: a systematic review and meta-analysis of their determinants and their association with cardiovascular events. Atherosclerosis. 2015;239(1):11-20.

10. Shah N, Chainani V, Delafontaine P, Abdo A, Lafferty J, Abi Rafeh N. Mammographically detectable breast arterial calcification and atherosclerosis. Cardiol Rev. 2014;22(2):69-78.

11. Detrano R, Guerci AD, Carr JJ, Bild DE, Burke G, Folsom AR, Liu K, et al. Coronary calcium as a predictor of coronary events in four racial or ethnic groups. N Engl J Med. 2008;358(13):1336-1345.

12. Taylor AJ, Bindeman J, Feuerstein I, Le T, Bauer K, Byrd $\mathrm{C}, \mathrm{Wu} \mathrm{H}$, et al. Community-based provision of statin and aspirin after the detection of coronary artery calcium within a community-based screening cohort. J Am Coll Cardiol. 2008;51(14):1337-1341.

13. Pecchi A, Rossi R, Coppi F, Ligabue G, Modena MG, Romagnoli R. Association of breast arterial calcifications detected by mammography and coronary artery calcifications quantified by multislice CT in a population of postmenopausal women. Radiol Med. 2003;106(4):305-312.

14. Maas AH, van der Schouw YT, Beijerinck D, Deurenberg JJ, Mali WP, van der Graaf Y. Arterial calcifications seen on mammograms: cardiovascular risk factors, pregnancy, and lactation. Radiology. 2006;240(1):33-38.

15. Matsumura ME, Maksimik C, Martinez MW, Weiss M, Newcomb J, Harris K, Rossi MA. Breast artery calcium noted on screening mammography is predictive of high risk coronary calcium in asymptomatic women: a case control study. Vasa. 2013;42(6):429-433.

16. Newallo D, Meinel FG, Schoepf UJ, Baumann S, De Cecco CN, Leddy RJ, Vliegenthart R, et al. Mammographic detection of breast arterial calcification as an independent predictor of coronary atherosclerotic disease in a single ethnic cohort of African American women. Atherosclerosis. 2015;242(1):218-221.

17. Chadashvili T, Litmanovich D, Hall F, Slanetz PJ. Do breast arterial calcifications on mammography predict elevated risk of coronary artery disease? Eur J Radiol. 2016;85(6):1121-1124.

18. Margolies L, Salvatore M, Hecht HS, Kotkin S, Yip R, Baber U, Bishay V, et al. Digital mammography and 
screening for coronary artery disease. JACC Cardiovasc Imaging. 2016;9(4):350-360.

19. Shaw LJ, Iskandrian AE. Prognostic value of gated myocardial perfusion SPECT. J Nucl Cardiol. 2004;11(2):171185.

20. Sager SJ, Hendel RC. Appropriate use of radionuclide imaging. Cardiol Rev. 2012;20(1):33-37.

21. Berman DS, Wong ND, Gransar H, Miranda-Peats R, Dahlbeck J, Hayes SW, Friedman JD, et al. Relationship between stress-induced myocardial ischemia and atherosclerosis measured by coronary calcium tomography. J Am Coll Cardiol. 2004;44(4):923-930.

22. Fathala A, Al Amer A, Shukri M, Abouzied MM, Alsugair A. The relationship between coronary artery calcification and myocardial perfusion in asymptomatic women. Ann Saudi Med. 2012;32(4):378-383.

23. Henzlova MJ, Duvall WL, Einstein AJ, Travin MI, Verberne HJ. ASNC imaging guidelines for SPECT nuclear cardiology procedures: Stress, protocols, and tracers. J Nucl Cardiol. 2016;23(3):606-639.

24. Zgheib MH, Buchbinder SS, Abi Rafeh N, Elya M, Raia C, Ahern K, Smith MC, et al. Breast arterial calcifications on mammograms do not predict coronary heart disease at coronary angiography. Radiology. 2010;254(2):367-373.

25. Moradi M, Adibi A, Abedi M. Relationship between breast arterial calcification on mammography with CT Calcium scoring and coronary CT angiography results. Adv Biomed Res. 2014;3:79.

26. Nakano T, Ninomiya T, Sumiyoshi S, Fujii H, Doi Y, Hirakata $H$, Tsuruya $K$, et al. Association of kidney function with coronary atherosclerosis and calcification in autopsy samples from Japanese elders: the Hisayama study. Am J Kidney Dis. 2010;55(1):21-30.

27. Bhatt DL. Anti-inflammatory agents and antioxidants as a possible "third great wave" in cardiovascular secondary prevention. Am J Cardiol. 2008;101(10A):4D-13D.

28. Reddy J, Son H, Smith SJ, Paultre F, Mosca L. Prevalence of breast arterial calcifications in an ethnically diverse population of women. Ann Epidemiol. 2005;15(5):344350 .

29. Henkin Y, Abu-Ful A, Shai I, Crystal P. Lack of association between breast artery calcification seen on mammography and coronary artery disease on angiography. J Med Screen. 2003;10(3):139-142. 